\title{
Association between insulin resistance and estrogen in sexual precocity of obese children
}

\author{
SHIXIA LIN ${ }^{1,2}$ and WEI JI ${ }^{2}$ \\ ${ }^{1}$ Department of Paediatrics, Jiangyin Hospital Affiliated to Medical School of Southeast University, Jiangyin, Jiangsu 214499; \\ ${ }^{2}$ Department of Paediatrics, Children's Hospital Affiliated to Soochow University, Suzhou, Jiangsu 215003, P.R. China
}

Received January 14, 2016; Accepted June 28, 2016

DOI: $10.3892 /$ etm.2016.3663

\begin{abstract}
The aim of the study was to examine the association between sexual precocity and high-molecular weight (HMW)-adiponectin and investigate the correlation of insulin resistance and estrogen levels in obese children. In total, 60 obese children ( 30 boys and 30 girls) with sexual precocity were included in group A, 60 obese children (30 boys and 30 girls) without sexual precocity were included in group B, and 60 average weight children ( 30 boys and 30 girls) were included in group C. The levels of HMW adiponectin, fasting blood glucose, fasting insulin, luteinizing hormone $(\mathrm{LH})$ peak, estradiol and testosterone were measured. The results showed that the HMW-adiponectin level of group A was the lowest and that of group $\mathrm{C}$ was the highest. The difference was statistically significant $(\mathrm{P}<0.05)$. The homeostasis model assessment of insulin resistance (HOMA-IR) and estradiol levels of group A were significantly higher than those of group B, and group B was higher than that of group C. LH peak and testosterone levels of group A were the lowest while those of group C were the highest. The differences were statistically significant $(\mathrm{P}<0.05)$. A subgroup analysis showed that the above results were more significant in girls. The Pearson correlation analysis revealed that the level of $\mathrm{HMW}$-adiponectin was negatively correlated with HOMA-IR and estradiol $(\mathrm{P}<0.05)$, and positively correlated with the LH peak $(\mathrm{P}<0.05)$. In conclusion, sexual precocity of obese children may be associated with insulin resistance, and the link may be HMW-adiponectin.
\end{abstract}

\section{Introduction}

The continuous improvement of the standard of living have led to an increase in the number of overweight and obese children

Correspondence to: Dr Wei Ji, Department of Pediatrics, Children's Hospital Affiliated to Soochow University, No. 303 Jing De Road, Suzhou, Jiangsu 215003, P.R. China

E-mail: ji_wei111@163.com

Key words: obesity, sexual precocity, insulin resistance, estrogen, high-molecular weight-adiponectin in China, and according to statistics, China's overweight and obese children in 2015 increased to 5 million (1). The phenomenon of precocious puberty caused by obesity has already aroused the concern of endocrine and nutrition experts, who suggest that the energy regulation disorder caused by obesity disrupts the regulatory pathway of normal gonadal development $(1,2)$. The two have a common neuroendocrine regulation factor and high-molecular weight (HMW)-adiponectin may play a key role as the 'bridge' (2). A related study suggested that obesity can cause the body to experience an insulin resistance phenomenon, reduce the use of sugar, fat, protein and other substances, leading to an insufficient energy supply, increasing the body's intake of more nutrients and energy, forming a vicious circle (3).

The specific mechanism underlying sexual precocity in obese children is not very clear and the effects of obesity between boys and girls are not exactly the same, and may even be opposite (4). Furthermore, the molecular basis of insulin resistance in obesity remains to be elucidated. However, whether there is a common substance between sexual maturity and insulin resistance remains to be determined. The aim of the study was to examine the association between sexual precocity and high-molecular weight (HMW)-adiponectin and investigate the correlation of insulin resistance and estrogen levels in obese children. The results showed that, sexual precocity of obese children may be associated with insulin resistance, and the link may be HMW-adiponectin.

\section{Materials and methods}

Materials. A total of 60 obese children (30 boys and 30 girls) diagnosed with sexual precocity at the Children's Hospital Affiliated to Soochow University between October, 2012 and October, 2015 were included in group A. Boys had an average age of $7.5 \pm 1.6$ and $7.0 \pm 1.5$ years for girls. The average body mass index (BMI) of $27.6 \pm 2.4 \mathrm{Kg} / \mathrm{m}^{2}$. During the same period, 60 obese children (30 boys and 30 girls) without sexual precocity were included in group B, with an average age of $7.6 \pm 1.8$ years and average BMI of $23.1 \pm 1.9 \mathrm{Kg} / \mathrm{m}^{2}$, and 60 normal weight children ( 30 boys and 30 girls) were included in group $\mathrm{C}$, with an average age of $7.8 \pm 1.7$ years and average BMI of $16.2 \pm 2.2 \mathrm{Kg} / \mathrm{m}^{2}$. There were no significant differences in gender and age among the three groups $(\mathrm{P}>0.05)$ and there was no significant difference in BMI between groups $\mathrm{A}$ and 
B ( $\mathrm{P}>0.05)$. Any subjects with inherited metabolic diseases, birth defects, and other related endocrine system diseases were excluded. Obesity was defined as BMI $\geq$ the 95th percentile of children of the same gender and age. Sexual precocity was defined as the appearance of secondary sexual characteristics in boys $<9$ years old and girls $<8$ years old, or the appearance of menstruation in girls $<10$ years old. B ultrasound identified an ovarian volume of $>1 \mathrm{ml}$, multiple foliculi (diameter $>4 \mathrm{~mm}$ ), boys' testicular volume $\geq 4 \mathrm{ml}$, gonadotrophin releasing hormone $(\mathrm{GnRH})$ provocative test showed luteinizing hormone (LH) $>5 \mathrm{IU} / 1$, and LH/follicle-stimulating hormone (FSH) $>0.6$ (chemiluminescence method).

Detection indexes. Approval for the study was obtained from the Ethics Committee of the Children's Hospital Affiliated to Soochow University. Written informed consent was obtained from the children's guardians.

Height and weight of the children were measured, the secondary sexual characteristics were diagnosed, the levels of serum HMW-adiponectin, fasting blood glucose (FPG), fasting insulin (FINS), LH peak, as well as estradiol and testosterone were detected. Height and weight were measured using the standard method 3 times, accurate to $0.1 \mathrm{~kg}$ and $0.1 \mathrm{~cm}$, and an average was obtained. Assessment of the secondary sexual characteristics including breast, pubic hair, testis, and penile development was made with the expertise of a special pediatric endocrine doctor. Breast development was evaluated by visual examination and palpation, and pubic hair development was evaluated by visual examination. According to the Tanner staging criteria, breast development was divided into B1-B5 stage, and the more mature development was recorded in the case that the breast development of the two sides was different. Pubic hair development was divided into PH1-PH5 stage, testicular volume was determined using the Rade testis model comparison method and an average was obtained for the two sides.

Detection methods. FINS was detected using a Siemens centuar XP chemiluminescence immunoassay analyzer (Siemens AG, Munich, Germany), FPG was detected using a Siemens ADVIA 2400 automatic biochemical analyzer (Siemens AG), and insulin resistance index was calculated as [homeostasis model assessment of insulin resistance (HOMA-IR)] $=($ FINS $) \times$ FPG/22.5. HMW-adiponectin was detected using a radio-immunity kit provided by Beijing Northern Biotechnology Research Institute (Beijing, China), and the serum antigens were detected according to the principle of homogeneous competition mechanism and balance competition method (2). The antigens in the samples and ${ }^{125} 1$-labeled antigens competitively formed immune reactions with limited specific antibodies. A high concentration of the antigen in the sample led to the labeled antigen bound to the antibody being decreased, whereas a low concentration of antigen in the sample led to the labeled antigen bound to the antibody being increased. The uncombined antigens were removed using an immune separation agent and the radioactivity of the compound was detected. The binding capacity of the labeled antigen was in a functional relationship with the antigen in the sample, thus, the concentration of the antigen in the sample could be determined through mathematical formulae.
LH peak was detected using the GnRH excitation test, GnRH [name: gonadorelin, batch no. H10960064; provided by BBCA Pharmaceutical Co., Ltd (Anhui, China)], $2.5-3 \mu \mathrm{g} / \mathrm{kg}$, maximum of $100 \mu \mathrm{g}$, and serum specimen were collected 30, 45, 60 and 90 min after injection and LH peak was detected.

The LH, estradiol and testosterone levels were detected using chemiluminescence. The LH ELISA kit (St. Louis, MO, USA; catalog no. SE120071), estradiol ELISA kit (Fitzgerald, NY, USA; catalog no. 55R-IB79301), and testosterone ELISA kit (Biovision, NY, USA; catalog no. K7417-100) were used to detect the levels of these hormones. Details steps were carried out according to the manufacturer's protocol.

Statistical analysis. SPSS 19.0 statistical software (SPSS, Inc., Chicago, IL, USA) was used for statistical analysis. Measurement data were presented as mean \pm standard deviation and the group comparison was made using analysis of variance. Countable data were expressed as a percentage (\%) and the group comparison was made using the $\chi^{2}$ test. A correlation analysis was performed using the Pearson correlation analysis. $\mathrm{P}<0.05$ was considered to indicate a statistically significant difference.

\section{Results}

Comparison of HMW-adiponectin and insulin resistance index. The HMW-adiponectin level of group A was the lowest and that of group $\mathrm{C}$ was the highest, and the difference was statistically significant $(\mathrm{P}<0.05)$. The insulin resistance index of group A was significantly higher than that of group B, and group B was higher than that of group C, with the differences being statistically significant $(\mathrm{P}<0.05)$. The subgroup analysis revealed that the above analysis was more significant in girls (Table I).

Comparison of LH peak, estradiol and testosterone levels. The LH peak value and testosterone levels of group A were the lowest and those of group $\mathrm{C}$ were the highest, with the difference being statistically significant $(\mathrm{P}<0.05)$. The estradiol level of group A was significantly higher than that of group B, and group B was higher than that of group $\mathrm{C}$, with the difference being statistically significant $(\mathrm{P}<0.05)$. The subgroup analysis revealed that the above differences were more significant in girls (Table II).

Correlation analysis. The Pearson correlation analysis of group A revealed that the level of HMW-adiponectin was negatively correlated with HOMA-IR $(\mathrm{r}=0.532, \mathrm{P}<0.001)$ and estradiol $(\mathrm{r}=0.326, \mathrm{P}=0.035)$, positively correlated with LH peak $r=0.425, P=0.019)$, but not significantly correlated with the testosterone level.

\section{Discussion}

Previous findings have indicated that sexual precocity is affected by environmental and genetic factors (5). Obesity may be a major factor that causes the activation of sexual initiation, especially in girls (6). Obesity may directly stimulate secretion of the hypothalamus $\mathrm{GnRH}$ and result in central precocious 
Table I. Comparison of HMW-adiponectin and insulin resistance index.

\begin{tabular}{lccccccc}
\hline & \multicolumn{3}{c}{ HMW-adiponectin $(\mu \mathrm{g} / \mathrm{ml})$} & & \multicolumn{3}{c}{ Insulin resistance index } \\
\cline { 2 - 3 } Group & Overall & Girls & Boys & & Overall & Girls & Boys \\
\hline A & $2.8 \pm 2.6$ & $2.7 \pm 1.5$ & $2.9 \pm 1.2$ & & $1.7 \pm 0.4$ & $2.1 \pm 0.5$ & $1.5 \pm 0.3$ \\
B & $3.4 \pm 2.8$ & $3.5 \pm 1.7$ & $3.3 \pm 1.9$ & & $1.3 \pm 0.3$ & $1.8 \pm 0.3$ & $1.2 \pm 0.2$ \\
C & $3.9 \pm 2.3$ & $4.3 \pm 2.5$ & $3.6 \pm 2.2$ & & $0.8 \pm 0.2$ & $0.7 \pm 0.1$ & $0.8 \pm 0.3$ \\
F-value & 4.562 & 5.326 & 4.687 & & 4.725 & 5.521 & 4.836 \\
P-value & 0.039 & 0.028 & 0.036 & & 0.033 & 0.022 & 0.031 \\
\hline
\end{tabular}

Group A, obese children with sexual precocity; group B, obese children without sexual precocity; group C, normal weight children. HMW, high-molecular weight.

Table II. Comparison of LH peak, estradiol and testosterone levels.

\begin{tabular}{|c|c|c|c|c|c|c|c|}
\hline \multirow[b]{2}{*}{ Group } & \multirow[b]{2}{*}{ Girls } & \multicolumn{2}{|c|}{ LH peak $(\mathrm{mIU} / \mathrm{ml})$} & \multicolumn{2}{|c|}{ Estradiol (pg/ml) } & \multicolumn{2}{|c|}{ Testosterone (ng/ml) } \\
\hline & & Overall & Girls & Boys & Overall & Girls & Boys \\
\hline A & $5.5 \pm 2.3$ & $50.2 \pm 12.2$ & $83.5 \pm 18.9$ & $35.2 \pm 10.4$ & $0.19 \pm 0.03$ & $0.04 \pm 0.01$ & $0.69 \pm 0.15$ \\
\hline B & $8.7 \pm 2.9$ & $37.8 \pm 9.6$ & $52.6 \pm 15.4$ & $23.3 \pm 12.3$ & $0.32 \pm 0.05$ & $0.13 \pm 0.06$ & $0.74 \pm 0.16$ \\
\hline $\mathrm{C}$ & $13.2 \pm 3.5$ & $26.3 \pm 8.3$ & $37.5 \pm 10.3$ & $15.4 \pm 8.5$ & $0.45 \pm 0.06$ & $0.26 \pm 0.09$ & $0.88 \pm 0.13$ \\
\hline F-value & 6.302 & 6.325 & 6.423 & 5.967 & 6.925 & 7.421 & 6.123 \\
\hline P-value & $<0.001$ & $<0.001$ & $<0.001$ & $<0.001$ & $<0.001$ & $<0.001$ & $<0.001$ \\
\hline
\end{tabular}

Group A, obese children with sexual precocity; group B, obese children without sexual precocity; group C, normal weight children. LH, luteinizing hormone.

puberty (7). It was previously suggested that adolescent obese girls' LH secretion pulse frequency and peak were significantly lower than those of normal weight girls (8). Lee et al (9) also suggested that LH stimulation peak in boys with sexual precocity was negatively correlated with BMI. However, in another study, the results showed that the high BMI value of girls with sexual precocity was correlated with pubic hair, armpit hair anticipation and advanced bone age, but not correlated with the LH/FSH ratio (10). At present, the specific mechanism regarding the manner in which obesity regulates sexual precocity remains to be clarified.

Previous findings suggested that there was insulin resistance in children with armpit hair anticipation (11). Insulin resistance can lead to a glucose utilization disorder and result in hyperlipidemia, high blood pressure, abnormal body fat distribution, atherosclerosis, coronary heart disease and other metabolic disorders (11). GnRH analogues can negatively feedback the regulation mechanism and inhibit the secretion of gonadal hormone, retarding linear growth and greatly improving the final adult height (12). Follow up showed that GnRH analogues may lead to insulin resistance and imbalanced body fat distribution (13). At present, whether there is insulin resistance in children with precocious puberty remains to be determined. The results of the present study have shown that the HMW-adiponectin level of obese children with sexual precocity was significantly lower than that of the normal weight children. HOMA-IR and estradiol levels were significantly increased while LH peak and testosterone levels were significantly reduced, and these differences were statistically significant. The Pearson correlation analysis revealed that the HMW-adiponectin level was negatively correlated with HOMA-IR and estradiol and positively correlated with LH peak, indicating sexual precocity of obese children may be associated with insulin resistance, and HMW-adiponectin may serve as a connection.

HMW-adiponectin is an important cytokine secreted by adipose cells and is important in the pathogenesis of metabolic syndrome (14). Adiponectin can regulate glucose and lipid metabolism, increase insulin, inhibit glycogenesis, anti-inflammation and protect blood vessels. Adiponectin can control the hypothalamus to decrease appetite and resist obesity, and increase insulin secretion and regulate the sensitivity of insulin in the target tissue. The study by Boyraz et al (15) showed that adiponectin level in obese children was significantly lower than that of normal-weight children of the same age. The mechanisms of adiponectin meditation in sexual precocity may be: i) Different from leptin, adiponectin was a peripheral cytokine. Reduced adiponectin levels can block energy utilization in the peripheral tissues; at this time, the central system would begin to consume the remaining energy (16). ii) The reduction of adiponectin and the accumulation of central system energy in turn can directly stimulate the secretion of gonadotropin releasing hormone and gonadotropin; in the model of rats, 
adiponectin receptors were observed in the hypothalamic neurons and pituitary gonadotropin releasing substances that caused kisspeptin. Adiponectin can accelerate the frequency of gonadotropin-releasing hormone and directly stimulate the release of progesterone and follicle hormones (17).

In addition, the subgroup analysis revealed that the above differences were more significant in girls than boys. The obesity of girls may be more closely associated with sexual hormone disturbance and obese girls were more prone to precocious puberty, whereas obese boys, due to their significantly reduced testosterone levels, may experience precocious puberty (18). In conclusion, the sexual precocity of obese children may be associated with insulin resistance, and HMW-adiponectin may serve as a link.

\section{Acknowledgements}

The present study was supported by the National Natural Science Foundation of China (grant no. 81570016).

\section{References}

1. Zhao YL, Ma RM, Lao TT, Chen Z, Du MY, Liang K, Huang YK, Zhang L, Yang MH, Sun YH, et al: Maternal gestational diabetes mellitus and overweight and obesity in offspring: a study in Chinese children. J Dev Orin Health Dis 6: 479-484, 2015.

2. Prodam F, Roccio M, Trovato L, Ricotti R, Moia S, Giglione E, Petri A, Walker GE, Bellone S and Bona G: Adiponectin oligomers are similarly distributed in adequate-for-gestational-age obese children irrespective of feeding in their first year. Pediatr Res 77: 808-813, 2015 .

3. LunegovaOS,Kerimkulova AS,Mjrrakhimov AE,Zalesskaya YV, Nabiev MP, Abilova SS, Neronova KV, IsakovaZhT, Aldashev AA and Mirrakhimov EM: An association between t455c polymorphism of apolipoprotein c-iii gene, insulin resistance and metabolic syndrome components. Kardiologiia 55: 47-53, 2015 (In Russian).

4. Eugster EA: Peripheral precocious puberty: Causes and current management. Horm Res 71 (Suppl 1): 64-67, 2009.

5. Euling SY, Selevan SG, Pescovitz OH and Skakkebaek NE: Role of environmental factors in the timing of puberty. Pediatrics 121 (Suppl 3): S167-S171, 2008.
6. Wronka I: Association between BMI and age at menarche in girls from different socio-economic groups. Anthropol Anz 68: 43-52, 2010.

7. Kaplowitz PB: Link between body fat and the timing of puberty. Pediatrics 121 (Suppl 3): S208-S217, 2008.

8. Collins JS, Beller JP, Burt Solorzano C, Patrie JT, Chang RJ, Marshall JC and McCartney CR: Blunted day-night changes in luteinizing hormone pulse frequency in girls with obesity: The potential role of hyperandrogenemia. J Clin Endocrinol Metab 99: 2887-2896, 2014.

9. Lee HS, Park HK, Ko JH, Kim YJ and Hwang JS: Impact of body mass index on luteinizing hormone secretion in gonadotropin-releasing hormone stimulation tests of boys experiencing precocious puberty. Neuroendocrinology 97: 225-231, 2013.

10. Giabicani E, Allali S, Durand A, Sommet J, Couto-Silva AC and Brauner R: Presentation of 493 consecutive girls with idiopathic central precocious puberty: A single-center study. PLoS One 8: e70931, 2013

11. Pilia S, Casini MR, Foschini ML, Minerba L, Musiu MC, Marras V, Civolani P and Loche S: The effect of puberty on insulin resistance in obese children. J Endocrinol Invest 32: 401-405, 2009.

12. Taşcilar ME, Bilir P, Akinci A, Köse K, Akçora D, Inceoğlu D and Fitöz SO: The effect of gonadotropin-releasing hormone analog treatment (leuprolide) on body fat distribution in idiopathic central precocious puberty. Turk J Pediatr 53: 27-33, 2011.

13. Chiocca E, Dati E, Baroncelli GI, Mora S, Parrini D, Erba P and Bertelloni S: Body mass index and body composition in adolescents treated with gonadotropin-releasing hormone analogue triptorelin depot for central precocious puberty: data at near final height. Neuroendocrinology 89: 441-447, 2009.

14. Maggio MC, Montaperto D, Maringhini S, Corrado C, Gucciardino E and Corsello G: Adiponectin, resistin and leptin in paediatric chronic renal failure: correlation with auxological and endocrine profiles. J Nephrol 27: 275-279, 2014.

15. Boyraz M, Cekmez F, Karaoglu A, Cinaz P, Durak M and Bideci A: Serum adiponectin, leptin, resistin and RBP4 levels in obese and metabolic syndrome children with nonalcoholic fatty liver disease. Biomark Med 7: 737-745, 2013.

16. Sevim DG and Kiratli H: Serum adiponectin, insulin resistance, and uveal melanoma: clinicopathological correlations. Melanoma Res: Dec 1, 2015 (Epub ahead of print).

17. Donoso MA, Muñoz-Calvo MT, Barrios V, Martínez G, Hawkins F and Argente J: Increased leptin/adiponectin ratio and free leptin index are markers of insulin resistance in obese girls during pubertal development. Horm Res Paediatr 80: 363-370, 2013.

18. Laron Z and Wang Y: Is obesity associated with early sexual maturation? Pediatrics 113: 171-172; author reply 171-172, 2004. 